\title{
New Perspectives in the Treatment of Neurocritical Patient
}

\author{
Luis Alberto Camputaro* \\ Intensive Care Division, University of Buenos Aires, Argentina
}

*Corresponding author: Luis Alberto Camputaro, University of Buenos Aires, Intensive Care Division, Coordinator Neurovascular Neurointensive Care, Hospital Juan A Fernandez, Buenos Aires, Argentina.

To Cite This Article: Luis Alberto Camputaro, New Perspectives in the Treatment of Neurocritical Patient. Am J Biomed Sci \& Res. 2020 - 7(1). AJBSR.MS.ID.001111. DOI: 10.34297/AJBSR.2020.07.001111.

Received: 眥 January 9, 2020; Published: 筒 January 24, 2020

\section{Opinion}

The vision of the Neurocritical patient has certainly changed in recent decades. This has allowed us better results in our patients from survival to the quality-quantity of sequelae.

What have been the reasons for this change, from our perspective?

1. We have managed to better categorize the risk from the diagnosis of neurological injury with more and better images (CT Scan, PET Scan, MRI, DSA, Tractography, etc.)

2. Our knowledge of pathophysiology has improved beyond primary damage. We know better the mechanisms that are triggered in each acute neurological injury causing secondary damage. From the different inflammatory mechanisms; alterations in Cerebral Blood Flow (FSC) due to loss of selfregulation, and its particular temporal behavior in each particular pathology [1,2]; to the intricate mechanisms of apoptosis. Thus, we have also generated new knowledge about the interrelationships that produce systemic impact in the face of the different Neurological insults [3].

3. The development of new technology allows us to visualize these pathophysiological phenomena, giving us more and better opportunities for therapeutic intervention $[4,5]$. Among others: Measurement of Intracranial Pressure (ICP) directly through various devices available or indirect through measurement of Optical Nerve Sheath Diameter (ONSD) or Transcranial Döppler (TCD); balance in brain oxygenation through Yugular Bulb, Near-Infrared Spectrometry or 02 Tissue Pressure PtiO2; electrical alterations through continuous EEG; metabolism alterations through Brain Micro-dialysis, etc.

4. The generation of Consensus and Guidelines allow us to use strategies with varied evidence, agreed between the different actors involved in the Neurocritical Patient treatment (Emergency Physicians, Neurologists, Neurosurgeons, Intensive Care Physicians, Cardiologists); met with the paramedical and nursing staf. This aspect has allowed us, beyond improving the results; to be able to exchange information in a more regulated and uniform manner.

But there is still a challenge to be left: to generate more Trained Human Resource for the Neurocritical Patient Treatment, as we must ensure that attention is done from these new perspectives from their first assistance [6].

Our challenge for the new generations, from a new vision; continue to improve the quality of care of our patients by impacting better results in a number of pathologies that year after year occupy more space in the concern of public health in our countries.

\section{References}

1. Martin NA, Patwardhan R, Alexander M, Africk C, Lee J, et al. (1997) DP Characterization of cerebral hemodynamic phases following severe head trauma: hypoperfusion, hyperemia, and vasospasm. J Neurosurg 87: 9-19.

2. Gebel JM, Jauch EC, Brott TG, Khoury J, Sauerbeck L, etc. (2002) Natural history of perihematomal edema in patients with hyperacute spontaneous intracerebral hemorrhage. Stroke 33(11): 2631-2635.

3. Mèloux A, Béjot Y, Rochette L, Cottin Y, Vergely C (2019) Brain-Heart interactions during ischemic Processes. Clinical and experimental evidences. Stroke.

4. Mazzeo AT, Gupta D (2018) Monitoring the injured brain. Journal of Neurosurgical Sciences 62(5): 549-562.

5. Stocchetti N, Carbonara M, Citerio G, Ercole A, Skrifvars M, et al. (2017) Severe traumatic brain injury: targeted management in the intensive care unit. Lancet Neurol 16: 452-464.

6. Camputaro L, Kovac A, Scalice L, Steinhaus M (2018) Who takes care of neurocritical patients in emergency departments? Exploration survey. J Neurol Stroke. 8(4): 223-226. 